\title{
Mask versus Prongs for Nasal Continuous Positive Airway Pressure in Preterm Infants: A Systematic Review and Meta-Analysis
}

\author{
Brian C. King ${ }^{a}$ Bheru B. Gandhi ${ }^{a} \quad$ Andrea Jackson ${ }^{b} \quad$ Lakshmi Katakam $^{a}$ \\ Mohan Pammi ${ }^{a}$ Gautham Suresh ${ }^{a}$ \\ a Section of Neonatology, Baylor College of Medicine, Houston, TX, USA; ${ }^{b}$ Outcomes and Impact Service, \\ Texas Children's Hospital, Houston, TX, USA
}

\section{Keywords}

Preterm infants · Respiratory distress syndrome .

Continuous positive airway pressure - Nasal mask ·

Bronchopulmonary dysplasia

\begin{abstract}
Nasal continuous positive airway pressure (NCPAP) is an effective method of respiratory support for preterm infants. Nasal masks and binasal prongs are two interfaces available to deliver NCPAP, and it is unclear if one is superior to the other. We conducted a systematic review and meta-analysis, using the methodology recommended by the Cochrane Collaboration, to compare the efficacy and safety of nasal masks versus binasal prongs to deliver NCPAP in preterm infants $<37$ weeks of gestation. Ovid MEDLINE, Embase, Scopus, the Cochrane database, and PubMed were searched in February 2019. Seven trials met the inclusion criteria. Among preterm infants requiring NCPAP, the use of a nasal mask, compared to nasal prongs, decreased the rate of NCPAP failure within $72 \mathrm{~h}$ (RR 0.72, 95\% Cl 0.53-0.97; number needed to treat for an additional beneficial outcome [NNTB] 12.5, 95\% Cl 7.1100; 5 trials, 576 participants; low-certainty evidence) and the incidence of nasal injury (RR 0.71, 95\% Cl 0.59-0.85; NNTB 8.3,
\end{abstract}

95\% Cl 5.6-16.7; 6 trials, 665 participants; low-certainty evidence). In a subgroup of preterm infants requiring NCPAP after resuscitation at birth, the use of a nasal mask decreased the incidence of moderate-to-severe bronchopulmonary dysplasia (RR 0.47, 95\% Cl 0.23-0.95; NNTB 16.7, 95\% Cl 9.1100; 4 trials, 395 participants; very-low-certainty evidence) and the need for subsequent surfactant administration (RR 0.78, 95\% Cl 0.64-0.96; NNTB 8.33, 95\% Cl 4.54-33.33; 4 trials, 395 participants; low-certainty evidence). The use of nasal masks for preterm infants requiring NCPAP was associated with a reduction in NCPAP failure, need for surfactant administration, and moderate-to-severe bronchopulmonary dysplasia (low- to very-low-certainty evidence). Given the potential clinical benefit and minimal risk associated with a change in patient interface, nasal masks should be considered the preferred interface for NCPAP delivery in preterm infants.

๑) 2019 S. Karger AG, Basel

\section{Introduction}

Nasal continuous positive airway pressure (NCPAP) is a simple, low-cost therapy that has become the standard method for providing noninvasive respiratory support in

\section{KARGER}

(c) 2019 S. Karger AG, Basel

E-Mail karger@karger.com

www.karger.com/neo
Brian C. King, MD

Newborn Center - West Tower 6-104

Texas Children's Hospital, 6621 Fannin Street

Houston, TX 77030 (USA)

E-Mail bcking@ texaschildrens.org 
premature infants $[1,2]$. NCPAP can be used as the initial method of respiratory support ("step-up" therapy), with intubation and mechanical ventilation reserved for infants where CPAP alone is insufficient. Early use of NCPAP in this manner has been shown to reduce the need for invasive ventilation and treatment with surfactants [3-5]. NCPAP can also be used as "step-down" therapy to provide respiratory support after extubation, where it has been shown to decrease the need for reintubation [6].

NCPAP devices have two primary components: a device to generate pressure and a patient interface used to deliver pressure. There are multiple options available for NCPAP interfaces. Prior systematic reviews comparing interfaces found that short binasal prongs reduced the risk of reintubation and had improved respiratory parameters when compared to single nasal prongs and nasopharyngeal prongs $[2,3,7]$. As a result, short binasal prongs are the most commonly used interface to deliver NCPAP.

Binasal prongs have significant limitations. In order to achieve effective delivery of CPAP, the prongs must be fitting tightly $[2,8]$. The force applied to accomplish this can lead to injury to the nares and nasal septum [2]. Additionally, there can be significant variability and difficulty in finding appropriately sized prongs for each infant.

The nasal mask was one of the first interfaces used to deliver CPAP in infants. Due to frequent nasal airway obstruction and difficulty maintaining an adequate seal, they quickly lost favor [7]. Newer masks have since been created, using three-dimensional facial scanning to create a more anatomically appropriate fit [8]. These newer nasal masks may be able to overcome earlier limitations by providing a better seal and minimizing airway obstruction. They may also provide a more even pressure distribution on the skin and minimize injury to the nares and nasal septum. Two systematic reviews have recently been published on this subject. The first compared the newer nasal masks to prongs with respect to nasal injury only, but it did not evaluate the efficacy of NCPAP delivery [9]. The second compared outcomes related to safety and efficacy, but it did not review all important secondary outcomes and did not analyze important subgroups [10]. New studies have been published since these systematic reviews were conducted that add to the body of evidence. Therefore, we performed this review with the primary objective of determining the safety and efficacy of NCPAP delivery via nasal masks, compared to binasal prongs, among preterm infants receiving NCPAP.

Nasal Mask versus Binasal Prongs in Preterm Infants

\section{Methods}

\section{Search Strategy}

Ovid MEDLINE, Embase, Scopus, the Cochrane database, and PubMed were searched (February 2019). The following websites were searched for ongoing trials: www.clinicaltrials.gov, www. controlled-trials.com, the Australian and New Zealand Clinical Trials Registry (http://www.anzctr.org.au), the World Health Organization (WHO) International Clinical Trials Registry (http:// www.who.int/ictrp/search/en/), and the Clinical Trials Registry of India (ctri.nic.in/clinicaltrials).

The database searches were conducted by a research librarian. The search for ongoing trials was independently conducted by two of the authors (B.C.K. and B.B.G.). The reference lists of relevant reviews on the topic were also searched to identify additional trials for inclusion. The specific search strategy is included in the supplementary information (for all online suppl. material, see www. karger.com/doi/10.1159/000496462). A review protocol was not registered for this study.

\section{Eligibility Criteria}

Study Types

Only randomized and quasi-randomized trials published in English after the year 2000 were included.

\section{Participants}

Studies using NCPAP in preterm infants ( $<37$ weeks of gestation) for the following indications were included: (1) immediately after delivery room resuscitation as the primary mode of respiratory support for the treatment of respiratory distress ("step-up" therapy) and (2) transitional respiratory support for infants who were extubated after a period of endotracheal mechanical ventilation ("step-down" therapy).

Intervention

Nasal mask (any brand) for the delivery of NCPAP.

Comparison

Binasal prongs (any brand) for the delivery of NCPAP.

\section{Outcomes}

Our primary outcomes of interest were (1) the proportion of infants requiring intubation and mechanical ventilation within 72 $h$ of initiation of NCPAP and (2) the proportion of infants with skin injury resulting from the NCPAP interface.

Our secondary outcomes were: (1) mortality; (2) the incidence of bronchopulmonary dysplasia (BPD) (defined as treatment with supplemental oxygen at 28 days for mild BPD and as treatment with supplemental oxygen at 36 weeks postmenstrual age for moderate-to-severe BPD); (3) retinopathy of prematurity (requiring treatment); (4) intraventricular hemorrhage (greater than grade 2); (5) pneumothorax; (6) days on NCPAP; and (7) surfactant use following randomization.

\section{Study Selection and Data Abstraction}

Three authors (B.C.K., B.B.G., and A.J.) independently reviewed the titles and abstracts of all original citations and determined the need for a full paper review based on the eligibility criteria. A consensus meeting was held to discuss any citations regarding which there were differences in determination between 
reviewers. A full paper review was then independently conducted by the same reviewers, and another consensus meeting was held to determine final inclusion. Study characteristics and outcome data were extracted independently by two of the authors (B.C.K. and B.B.G.) on a predesigned spreadsheet and compared for consistency after completion.

\section{Risk of Bias in Individual Trials}

Each study included in the review was assessed for risk of bias in seven domains, using the Cochrane risk-of-bias tool (suppl. information; [11]), and categorized as "high risk," "low risk," or "unclear." The seven domains are: (1) sequence generation, (2) allocation concealment, (3) blinding of participants and personnel, (4) blinding of outcome assessment, (5) incomplete outcome data, (6) selective outcome reporting, and (7) other sources of bias. Any discrepancies in risk assessment between the two authors' categorizations were resolved in a consensus meeting.

\section{Synthesis of Results and Statistical Analysis}

The unit of analysis was the participant in a randomized controlled trial or quasi-randomized controlled trial. The authors of included trials were all contacted for additional information for completeness of data. When possible, we examined outcomes among all study participants and conducted a subgroup analysis by CPAP indication: "step-up" therapy and "step-down" therapy.

We used the statistical package (RevMan 5.3) provided by the Cochrane Collaboration for the generation of systematic reviews and performance of meta-analyses. We analyzed treatment effects in individual trials. We reported risk ratios (RRs) and risk differences (RDs) with 95\% confidence intervals (CIs) for dichotomous variables and weighted mean differences with $95 \%$ CIs for continuous variables. We calculated and reported the number needed to treat for an additional beneficial outcome (NNTB) for statistically significant differences in RD. We applied a fixed-effect model for meta-analysis when appropriate. For estimates of typical RR and RD, we used the Mantel-Haenszel method (denoted as RR and $\mathrm{RD}$ when summarizing results from the meta-analysis).

We assessed heterogeneity of treatment effects between trials using the $I^{2}$ statistic. If we detected statistical heterogeneity, we explored possible causes including differences in study quality, participants, intervention regimens, or outcome assessments.

\section{Certainty of Evidence Assessment}

We used the Grading of Recommendations, Assessment, Development and Evaluation (GRADE) [12, 13] approach to categorize the certainty of evidence for each clinically relevant outcome as: (1) high - "we are very confident that the true effect lies close to the estimate of the effect"; (2) moderate - "the true effect is likely to be close to the estimate of the effect, but there is a possibility that it is substantially different"; (3) low - "the true effect may be substantially different from the estimate of the effect"; or (4) very low - "the true effect is likely to be substantially different from the estimate of effect."

Two review authors independently assessed the certainty of the evidence for each outcome. We considered evidence from randomized controlled trials as "high certainty," but downgraded the evidence 1 level for serious (or 2 levels for very serious) limitations in the following domains: design (risk of bias), consistency across studies, directness of the evidence, precision of estimates, and for the presence of publication bias.

\section{Results}

\section{Search Results}

Our search strategy identified 7 randomized controlled trials that met the eligibility criteria (Fig. 1). We reviewed the full text of 5 additional studies that appeared potentially eligible based on review of the title and abstract, but we excluded them for the following reasons: lack of comparison between interfaces [14], single-author editorial review [15], observational study [16], did not include nasal masks [17], and limitation in language translation [18]. Our search strategy did not identify any randomized controlled trials published prior to the year 2000 that would have otherwise met our eligibility criteria.

Two ongoing trials were identified that met the eligibility criteria. Both trials were registered and documented as completed, with no published report of outcomes available $[19,20]$. The authors of these trials were contacted twice with no response.

\section{Study Characteristics}

A summary of the included trials is presented in Table 1. All identified trials on this topic included only preterm infants with a gestational age at birth that ranged from 26 to 34 weeks and/or very-low-birth weight infants $(<1,500$ g). Three trials were conducted in India $[1,21,22]$ and 1 each in the USA [23], Malaysia [24], the Republic of Ireland [5], and Turkey [8].

Four of the trials exclusively randomized patients requiring NCPAP as "step-up" therapy $[1,8,21,22]$. One trial randomized patients treated with NCPAP as "step-up" therapy and "step-down" therapy, and reported outcomes for each subgroup [5]. The remaining 2 trials randomized patients needing NCPAP for either indication and did not separate them when reporting outcomes [23, 24].

In all but one trial, the NCPAP interface to which patients were randomized was maintained in place until NCPAP was no longer required, or until the infant required intubation and mechanical ventilation. One trial initially randomized patients to nasal mask and nasal prongs, and then after $8 \mathrm{~h}$ conducted a second randomization from each group to create a third group rotating between interfaces [21]. For the meta-analysis, only patients who remained in the nasal mask or nasal prong groups were included in our analysis. Another trial had a third group randomized to alternating interfaces, but this group was not included in our meta-analysis [23]. The included trials used a variety of NCPAP devices for pressure generation, a variety of levels of CPAP, and various commercial brands of NCPAP systems (Table 1). 
Fig. 1. PRISMA flow diagram for search re-

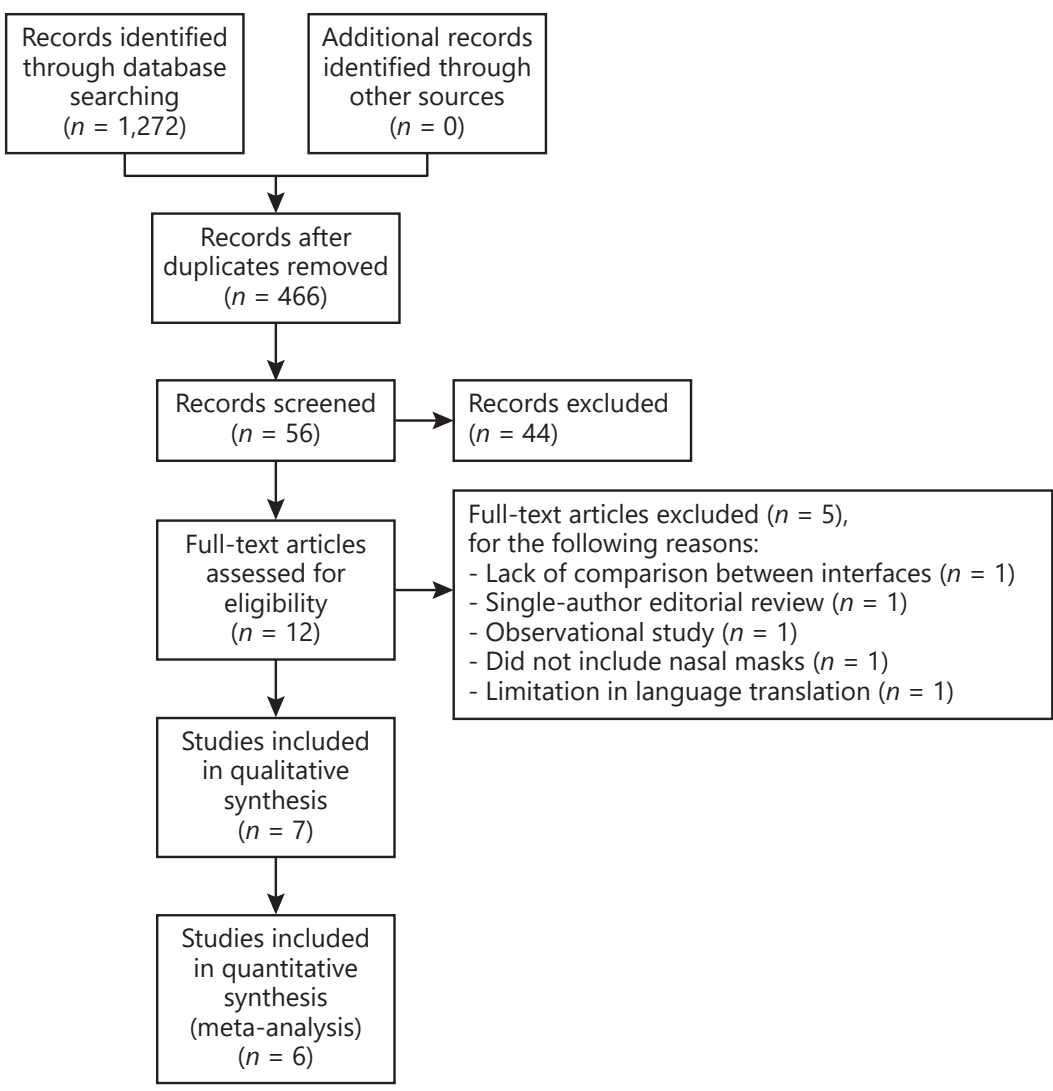
sults.

\section{Reporting of Outcomes}

Five trials reported data on NCPAP failure, defined as the need for mechanical ventilation within $72 \mathrm{~h}$ of NCPAP initiation. Each trial had explicit criteria for failure, which are summarized in Table 1 . One trial included nasal intermittent positive pressure ventilation as an option for support prior to intubation and mechanical ventilation, but it did not describe the clinical criteria used to determine the need for nasal intermittent positive pressure ventilation [5]. In extracting data on NCPAP failures from this trial, we only included infants needing intubation. One trial reported NCPAP failure at multiple time points following initiation of NCPAP [8]. In extracting data from this trial, we only used data on NCPAP failure within $72 \mathrm{~h}$ of treatment initiation.

All 7 trials reported data on nasal injury. Each trial used a different methodology and scoring system to define injury (Table 1). Three trials graded nasal injury as mild, moderate, or severe $[1,21,22]$, though 1 of those trials reported patient data for only the moderate cate- gory [21]. An additional trial did not report a grading scheme but provided descriptions of nasal injury for all patients [24]. For that trial, injury was assigned in keeping with the criteria from other included trials and based on the following criteria: mild - erythema/tenderness; moderate - excoriation/crusting/bleeding; and severe - narrowing of the nasal passage. Two trials did not include any grading scheme $[5,8]$, and a third trial used a grading scheme that did not allow for a clear separation into mild, moderate, and severe [23].

Five trials reported data on surfactant administration. In 2 trials, infants could receive surfactants prior to randomization and there were no differences in pre-randomization surfactants between groups in either trial [5, 22]. Four trials used an endotracheal tube for administration $[1,5,21,22]$, whereas 1 trial used a less-invasive strategy [8]. Endotracheal intubation for surfactant administration was not considered an NCPAP failure in any trial, unless an infant met the explicit clinical criteria for failure (Table 1). Three of the trials reported explicit criteria for 


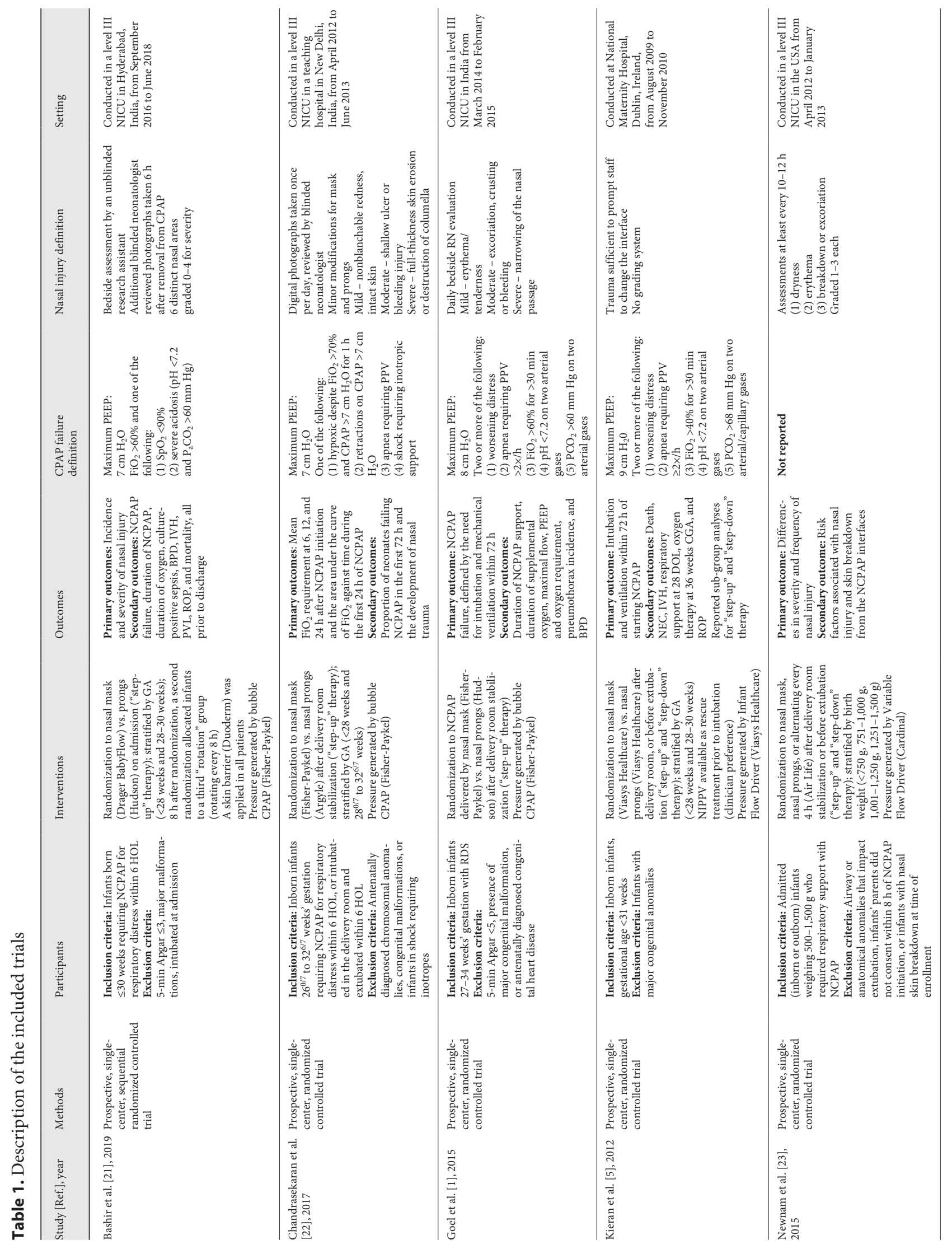




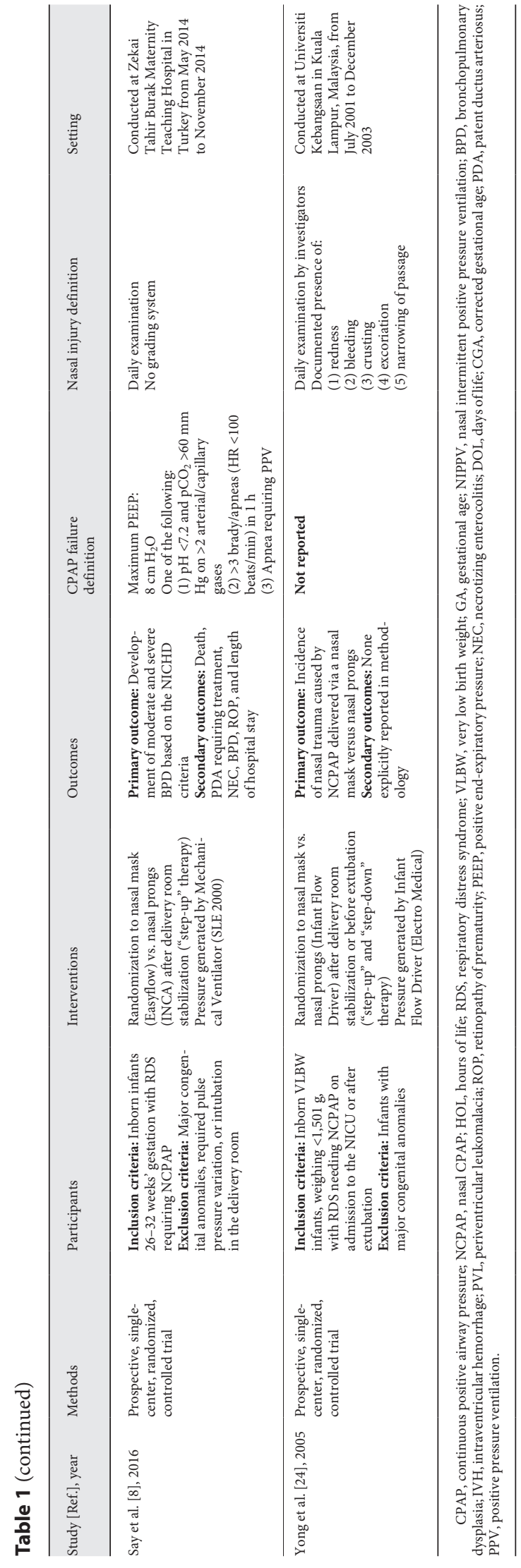

Nasal Mask versus Binasal Prongs in Preterm Infants

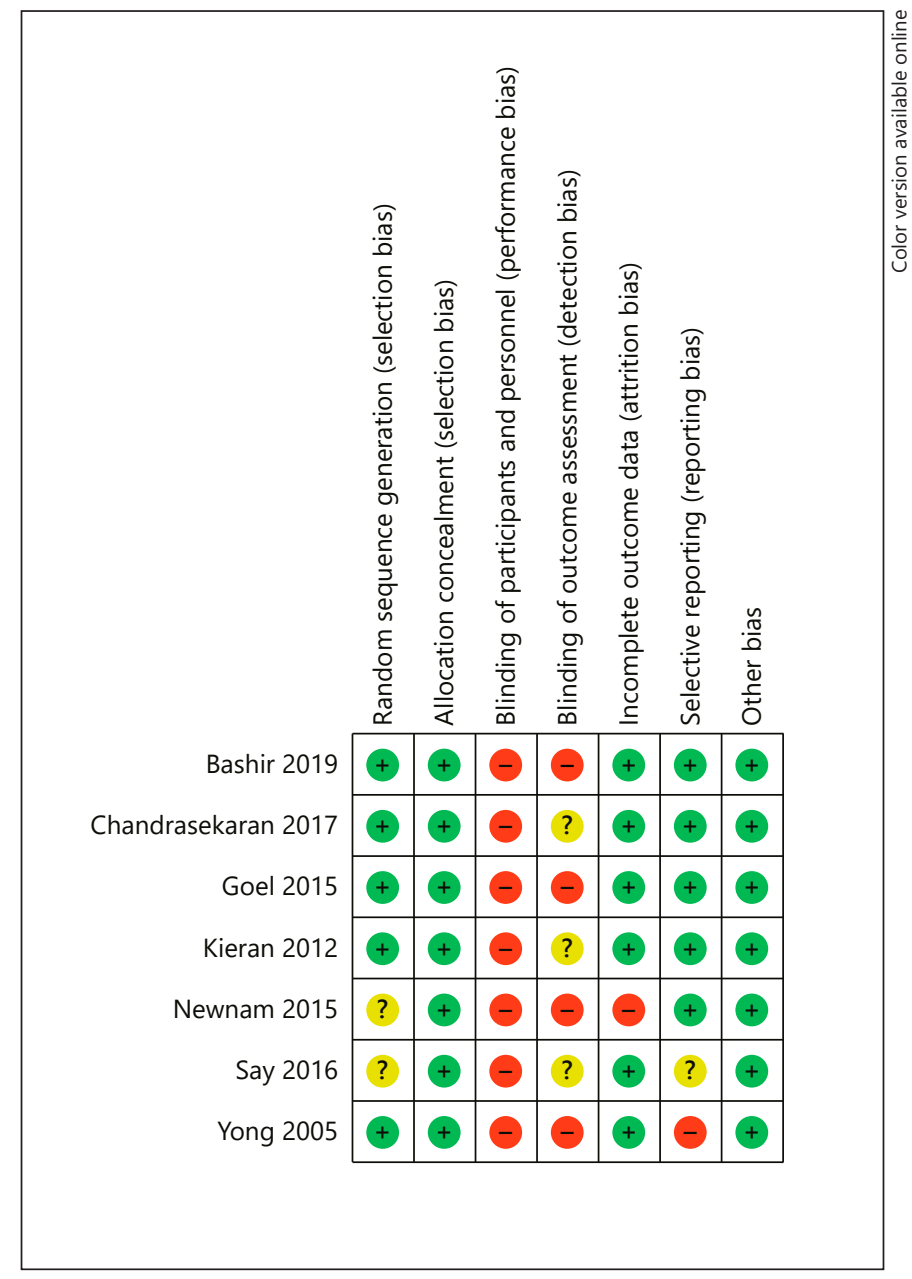

Fig. 2. Risks of bias in the included trials. Green indicates a low risk of bias, yellow indicates an unclear risk of bias (inability to evaluate based on reported methodology), and red indicates a high risk of bias.

surfactant administration $\left(\mathrm{FiO}_{2}\right.$ requirement), while 2 trials did not $[5,21]$.

In response to our requests for additional unpublished data, the authors of 2 trials provided data regarding duration of NCPAP $[8,22]$, and the authors of 1 trial provided data on their subgroup analysis [5]. The other authors were contacted 3 times each with no response. One trial did not include any data that could be extracted for primary or secondary outcomes, so it was not included in the meta-analysis [23].

\section{Risk of Bias}

Figure 2 summarizes the risk of bias for each included study. 
Randomization and Allocation Concealment

Five of the 7 trials described an adequate methodology for randomization and allocation concealment. Two trials did not clearly specify their methodology and were considered high risk $[8,23]$.

\section{Blinding of Intervention}

Due to the nature of the intervention, none of the trials were able to blind the clinicians to the type of interface used and were therefore all considered high risk.

\section{Blinding of Outcome Assessment}

One trial reported blinding the outcome assessors who reviewed photographs of nasal injury, but it did not report blinding for any other outcome; thus, it was considered unclear risk [22]. One trial similarly reported a blinded outcome assessor reviewing photographs of nasal injury, but also explicitly stated that other outcome assessments were not blinded; therefore, it was considered high risk [21]. The other trials either did not blind outcome assessment or were unclear regarding blinding of outcome assessment.

\section{Incomplete Outcome Data}

One trial defaulted patients who could not fit the binasal prongs into the "nasal mask" group and performed an as-treated analysis; it was therefore considered high risk [23]. One trial conducted a second randomization after $8 \mathrm{~h}$ to create a "rotation" group, but for our metaanalysis we only included infants who remained in their original group (nasal mask or binasal prongs), and so the trial was considered low risk [21]. The remaining trials had adequate follow-up and all patients were analyzed in their original group allocation.

\section{Selective Reporting}

One trial was considered high risk because the authors did not prespecify any secondary outcomes but reported results [24]. The authors of 1 trial stated that daily examinations had occurred for multiple types of nasal injury, but they reported only "skin breakdown" as their outcome; thus, the trial was considered unclear risk [8].

\section{Effects of Interventions}

Table 2 presents the summary of the findings for each outcome.

All Eligible Patients

NCPAP Failure: Need for Mechanical Ventilation within $72 \mathrm{~h}$ after NCPAP Initiation. Preterm infants random- ized to NCPAP delivered by nasal mask had a lower rate of NCPAP failure within the first $72 \mathrm{~h}$ after treatment initiation when compared to infants randomized to binasal prongs (RR 0.72, 95\% CI 0.53-0.97; RD -0.08, 95\% CI -0.14 to -0.01 ; NNTB 12.5 , 95\% CI $7.1-100 ; 5$ trials, 576 participants; low-certainty evidence) (Fig. 3). We downgraded the certainty of evidence to "low" because of the risks of bias and due to imprecision in the estimate of effect.

Incidence of Nasal Injury. Compared to infants receiving NCPAP by nasal prongs, preterm infants randomized to NCPAP delivered via nasal mask had a lower incidence of nasal injury (RR $0.71,95 \%$ CI $0.59-0.85$; RD $-0.12,95 \%$ CI -0.18 to -0.06 ; NNTB $8.3,95 \%$ CI $5.6-16.7$; 6 trials, 665 participants; very-low-certainty evidence) (Fig. 4). Preterm infants randomized to the nasal mask also had a lower incidence of moderate-to-severe nasal injury (RR 0.27 , $95 \%$ CI $0.16-0.46$; RD $-0.21,95 \%$ CI -0.28 to -0.14 ; NNTB 4.8, 95\% CI 3.6-7.1; 4 trials, 396 participants; lowcertainty evidence). The results show moderate heterogeneity ( $I^{2}=75$ and $83 \%$, respectively) among the 6 trials for this outcome (Fig. 3). We downgraded the certainty of evidence to "low" because of the risks of bias, and inconsistency of evidence.

Secondary Outcomes. There were no differences in the incidence of retinopathy of prematurity (requiring treatment), intraventricular hemorrhage (greater than grade 2 ), or pneumothorax, the duration of NCPAP (in days), and the incidence of BPD (all grades, as well as moderate to severe) and death before discharge between infants randomized to the nasal mask and those randomized to binasal prongs among all eligible patients (see online suppl. information).

\section{NCPAP as "Step-Up" Therapy}

NCPAP Failure: Need for Mechanical Ventilation within $72 \mathrm{~h}$ after NCPAP Initiation. In preterm infants receiving NCPAP as "step-up" therapy only, there was no significant difference in the rate of NCPAP failure within 72 $\mathrm{h}$ of randomization between infants randomized to the nasal mask and those randomized to binasal prongs (RR $0.78,95 \%$ CI $0.57-1.08$; RD $-0.05,95 \%$ CI -0.12 to 0.02 ; 5 trials, 513 participants; low-certainty evidence) (Fig. 3). We downgraded the certainty of evidence to "low" because of the risks of bias and due to imprecision in the estimate.

Incidence of Nasal Injury. Preterm infants requiring NCPAP for "step-up" therapy and randomized to the nasal mask had a lower incidence of nasal injury (RR 0.68, 95\% CI $0.56-0.83$; RD $-0.15,95 \%$ CI -0.22 to -0.09 ; 
Table 2. Summary of the findings for primary and secondary outcomes: NCPAP mask compared with NCPAP binasal prongs for preterm infants with respiratory distress

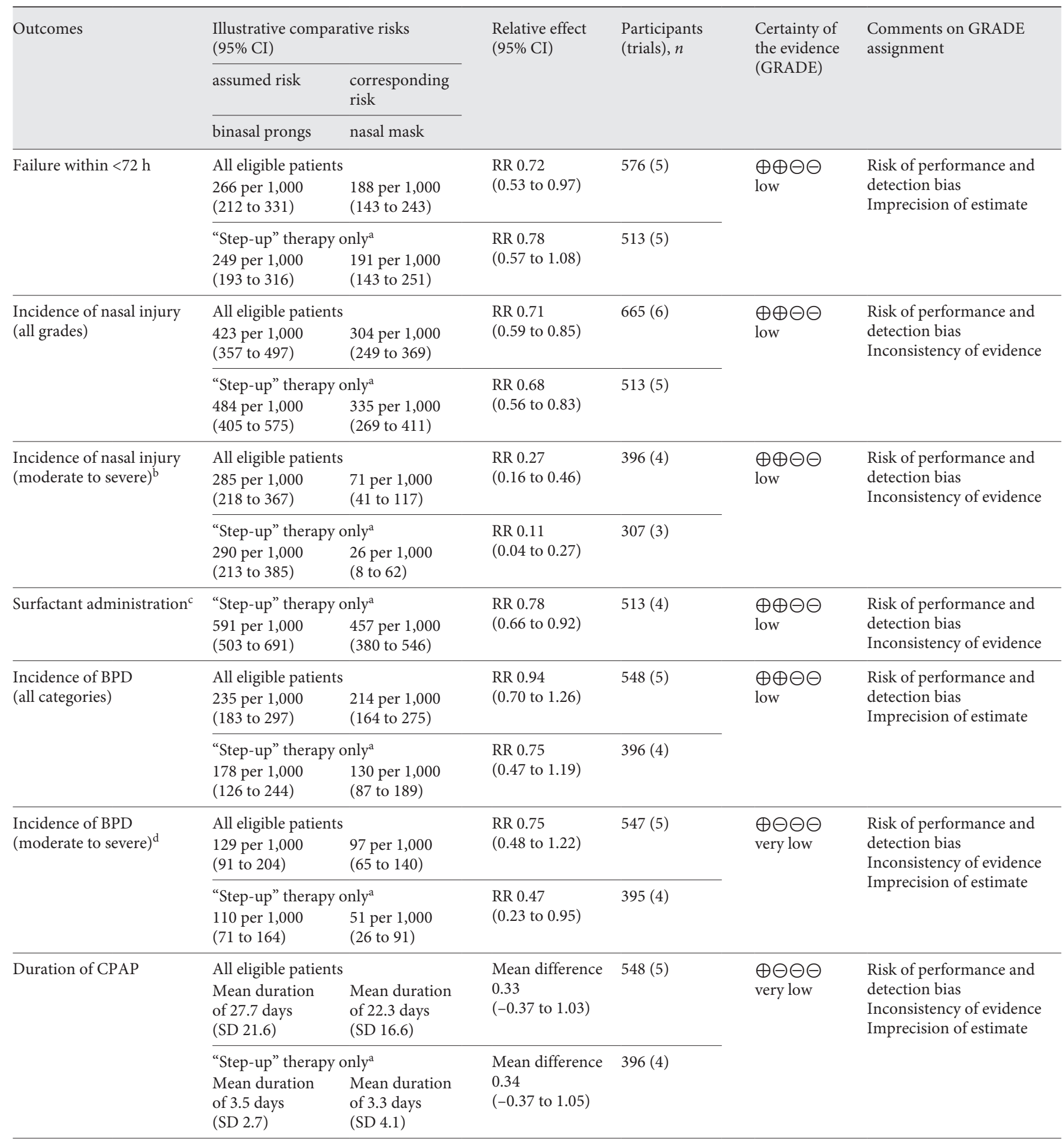


Table 2 (continued)

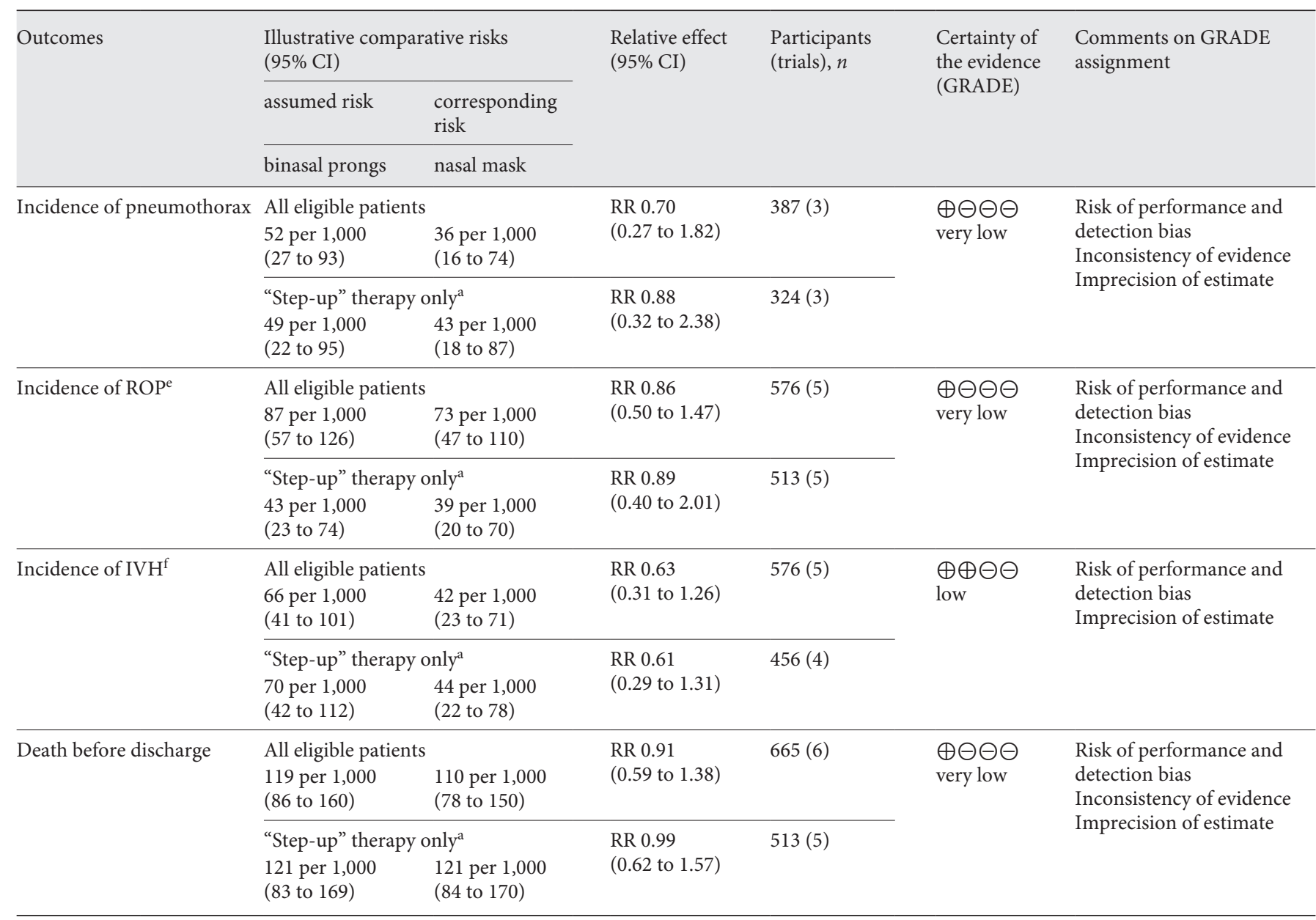

Patients or population: preterm infants requiring CPAP for initial respiratory support ("step-up" therapy) or after extubation ("step-down" therapy). Setting: Neonatal Intensive Care Units. Intervention: NCPAP - nasal mask. Comparison: NCPAP - binasal prongs. The corresponding risk (and its 95\% CI) is based on the assumed risk in the comparison group and the relative effect of the intervention (and its 95\% CI). GRADE Working Group grades of evidence - downgraded 1 level for each criterion listed in Comments. High certainty: further research is very unlikely to change our confidence in the estimate of the effect. Moderate certainty: further research is likely to have an important impact on our confidence in the estimate of the effect and may change the estimate. Low certainty: further research is very likely to have an important impact on our confidence in the estimate of the effect and is likely to change the estimate. Very low certainty: we are very uncertain about the estimate. CPAP, continuous positive airway pressure; NCPAP, nasal CPAP; CI, confidence interval; RR, risk ratio; BPD, bronchopulmonary dysplasia; ROP, retinopathy of prematurity; IVH, intraventricular hemorrhage. ${ }^{\text {a }}$ Infants randomized on admission to the Neonatal Intensive Care Unit after delivery room resuscitation. " "Moderate to severe defined" as excoriation, crusting, bleeding, or narrowing of the nasal passage. ${ }^{c}$ Surfactant administration after randomization, not including delivery room resuscitation. ${ }^{\mathrm{d}}$ Defined as the need for respiratory support at 36 weeks postmenstrual age. ${ }^{\mathrm{e}}$ Defined as ROP requiring treatment in 3 trials $[3,8,21]$, and stage $>2$ in 1 trial $[1] .{ }^{\mathrm{f}}$ Defined as IVH greater than grade 2 .

NNTB 6.7, 95\% CI 4.5-11.1; 5 trials, 513 participants; low-certainty evidence) (Fig. 4). Preterm infants randomized to the nasal mask for "step-up" therapy also had a lower incidence of moderate-to-severe nasal injury (RR $0.11,95 \%$ CI $0.04-0.27$; RD $-0.26,95 \%$ CI -0.34 to -0.19 ; NNTB 3.8, 95\% CI 2.9-5.3; 3 trials, 307 participants; lowcertainty evidence). The results show moderate heterogeneity for all grades of nasal injury $\left(I^{2}=87 \%\right)$ among the 5 trials for this outcome (Fig. 3). We downgraded the cer- tainty of evidence to "low" because of the risks of bias, and inconsistency of evidence.

Secondary Outcomes. In infants requiring NCPAP for "step-up" therapy, use of the nasal mask was associated with a lower incidence of moderate-to-severe BPD, defined as oxygen use at 36 weeks (RR 0.47, 95\% CI 0.230.95; RD -0.06, 95\% CI -0.11 to -0.01; NNTB 16.7, $95 \%$ CI 9.1-100; 4 trials, 395 participants; Fig. 5; very-low-certainty evidence). There was no difference in the incidence 


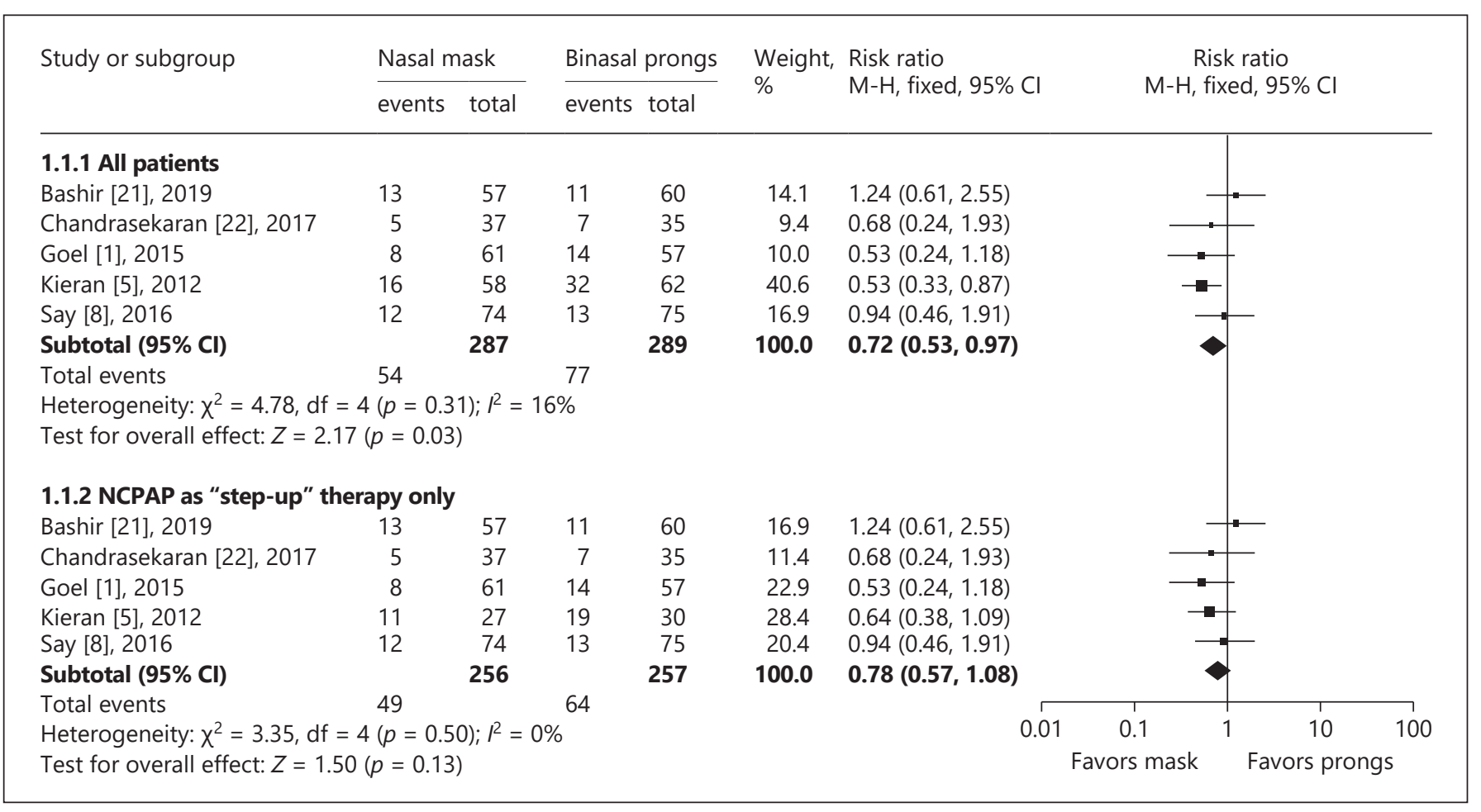

Fig. 3. Failure of NCPAP within $<72 \mathrm{~h}$ in all eligible patients, as well as in a subgroup of patients receiving NCPAP as "step-up" therapy only. NCPAP, nasal continuous positive airway pressure.

of BPD (all grades). We downgraded the certainty of the evidence to "very low" because of the risks of bias, inconsistency of evidence, and imprecision in the estimate. In infants requiring NCPAP for "step-up" therapy, use of the nasal mask was associated with a lower rate of surfactant administration after randomization (RR 0.78, 95\% CI $0.64-0.96$; RD $-0.12,95 \%$ CI -0.22 to -0.03 ; NNTB 8.33, 95\% CI 4.54-33.33; 4 trials, 395 participants; Fig. 5; low-certainty evidence). Three of the trials reported the incidence of surfactant administration prior to randomization, and there was no difference found between groups. We downgraded the certainty of the evidence to "low" because of the risks of bias, and inconsistency of evidence. There were no other significant differences identified in any other secondary outcomes among infants randomized to "step-up" therapy (see online suppl. information).

\section{NCPAP as "Step-Down" Therapy}

Only 1 trial randomized patients requiring NCPAP to both "step-up" and "step-down" therapy and reported outcomes for each subgroup [5]. In that trial, preterm in- fants receiving NCPAP as "step-down" therapy and randomized to the nasal mask had a lower rate of NCPAP failure within the first $72 \mathrm{~h}$ after treatment initiation when compared to infants randomized to binasal prongs (RR $0.40,95 \%$ CI $0.16-0.98$; RD $-0.24,95 \%$ CI -0.46 to -0.03 ; NNTB 4.17, 95\% CI 6.25-33.33; 1 trial, 63 participants; very-low-certainty evidence) [5]. There were no differences in nasal injury between the two groups, and no difference in any secondary outcomes reported (data not shown).

\section{Discussion}

This systematic review and meta-analysis addressed the safety and efficacy of NCPAP delivered via nasal masks compared to binasal prongs using two indications for NCPAP: when NCPAP is used as the primary respiratory support after delivery room resuscitation ("step-up" therapy) and when NCPAP is used for post-extubation respiratory support ("step-down" therapy). To our knowledge, this is the most up-to-date review on this top- 


\section{a All nasal injury}

Study or subgroup

Nasal mask events total $\frac{\text { Binasal prongs }}{\text { events total }}$
Weight, Risk ratio

$\mathrm{M}-\mathrm{H}$, fixed, 95\% Cl
Risk ratio

$\mathrm{M}-\mathrm{H}$, fixed, $95 \% \mathrm{Cl}$

\subsubsection{All patients}

Bashir [21], 2019

Chandrasekaran [22], 2017

Goel [1], 2015

Kieran [5], 2012

Say [8], 2016

Yong [24], 2005

Subtotal $(95 \% \mathrm{Cl})$

Total events

$\begin{array}{rr}19 & 57 \\ 30 & 37 \\ 22 & 61 \\ 2 & 58 \\ 15 & 75 \\ 12 & 41 \\ & \end{array}$

57
37
61
58
75
41
329

\section{5}

25

60

35

\section{0}

18.2

$0.36(0.25,0.53)$

$1.14(0.87,1.47)$

57

24.2

$0.62(0.42,0.93)$

$1.07(0.16,7.34)$

$7.1 \quad 1.48(0.71,3.08)$

$11.10 .83(0.45,1.52)$

Heterogeneity: $\chi^{2}=29.41, \mathrm{df}=5(p<0.0001) ; R^{2}=83 \%$

Test for overall effect: $Z=3.65(p=0.0003)$

\subsubsection{NCPAP as "step-up" therapy only}

$\begin{array}{lrrrr}\text { Bashir [21], 2019 } & 19 & 57 & 55 & 60 \\ \text { Chandrasekaran [22], 2017 } & 30 & 37 & 25 & 35 \\ \text { Goel [1], 2015 } & 22 & 61 & 33 & 57 \\ \text { Kieran [5], 2012 } & 0 & 27 & 1 & 30 \\ \text { Say [8], 2016 } & 15 & 75 & 10 & 74 \\ \text { Subtotal (95\% Cl) } & & \mathbf{2 5 7} & & \mathbf{2 5 6}\end{array}$

Total events $\quad 86 \quad 124$

Heterogeneity: $\chi^{2}=30.02, \mathrm{df}=4(p<0.00001) ; r^{2}=87 \%$

Test for overall effect: $Z=3.84(p=0.0001)$

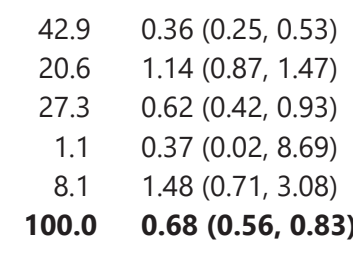

$100.0 \quad 0.68(0.56,0.83)$

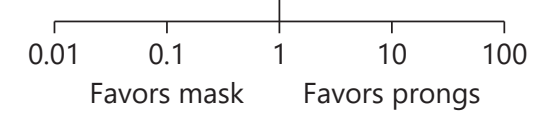

\section{b Moderate to severe nasal injury}

Study or subgroup

\section{Nasal mask}

$\underline{\text { Binasal prongs }}$

Weight, Risk ratio

$\% \quad \mathrm{M}-\mathrm{H}$, fixed, 95\% Cl

Risk ratio

$\mathrm{M}-\mathrm{H}$, fixed, $95 \% \mathrm{Cl}$

events total events total

\subsubsection{All patients}

Bashir [21], 2019

Chandrasekaran [22], 2017

Goel [1], 2015

Yong [24], 2005

Subtotal $(95 \% \mathrm{Cl})$

\%

Total events $14 \quad 57$

Heterogeneity: $\chi^{2}=15.41, \mathrm{df}=3(p=0.001) ; p^{2}=81 \%$

Test for overall effect: $Z=4.81(p<0.00001)$

\subsubsection{NCPAP as "step-up" therapy only}

\section{Bashir [21], 2019}

Chandrasekaran [22], 2017

Goel [1], 2015

Subtotal $(95 \% \mathrm{Cl})$

Total events

$\begin{array}{rrr}0 & 57 & 22 \\ 0 & 37 & 10 \\ 4 & 61 & 12 \\ & \mathbf{1 5 5} & \end{array}$

Heterogeneity: $\chi^{2}=5.30, \mathrm{df}=2(p=0.07) ; p^{2}=62 \%$

Test for overall effect: $Z=4.78(p<0.00001)$

$\begin{array}{rrr}60 & 38.4 & 0.02(0.00,0.38) \\ 35 & 18.9 & 0.05(0.00,0.74) \\ 57 & 21.7 & 0.31(0.11,0.91) \\ 48 & 21.0 & 0.90(0.44,1.83) \\ \mathbf{2 0 0} & \mathbf{1 0 0 . 0} & \mathbf{0 . 2 7}(\mathbf{0 . 1 6}, \mathbf{0 . 4 6})\end{array}$

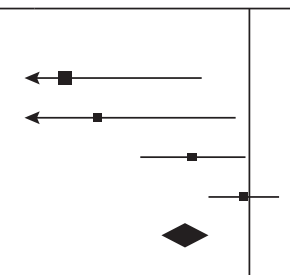

Fig. 4. Incidence of nasal injury in all eligible patients, as well as in a subgroup of patients receiving NCPAP as "step-up" therapy only. NCPAP, nasal continuous positive airway pressure. 
a Bronchopulmonary dysplasia

Study or subgroup

$$
\frac{\text { Nasal mask }}{\text { events total }}
$$

Binasal prongs

events total

Weight, Risk ratio

$\mathrm{M}-\mathrm{H}$, fixed, $95 \% \mathrm{Cl}$

Risk ratio

$\mathrm{M}-\mathrm{H}$, fixed, $95 \% \mathrm{Cl}$

1.4.1 All BPD (Oxygen at 28 days)

Chandrasekaran [22], $2017 \quad 6$

Goel [1], 2015

Kieran [5], 2012

Say [8], 2016

Subtotal $(95 \% \mathrm{Cl})$

Total events

$6 \quad 37$

Heterogeneity: $\chi^{2}=0.64, \mathrm{df}=3(p=0.89) ; R^{2}=0 \%$

Test for overall effect: $Z=1.22(p=0.22)$

\subsubsection{Moderate/severe BPD (oxygen at $\mathbf{3 6}$ weeks)}

Bashir [21], 2019

Chandrasekaran [22], 2017

157

Kieran [5], 2012

Say [8], 2016

Subtotal $(95 \% \mathrm{Cl})$

$3 \quad 37$

$4 \quad 27$

$2 \quad 74$

7

195

Heterogeneity: $\chi^{2}=4.28, \mathrm{df}=3(p=0.23) ; R^{2}=30 \%$

Test for overall effect: $Z=2.09(p=0.04)$

$\begin{array}{rrr}60 & 13.4 & 0.35(0.04,3.28) \\ 35 & 23.5 & 0.57(0.15,2.20) \\ 30 & 13.0 & 1.48(0.36,6.03) \\ 75 & 50.1 & 0.18(0.04,0.80) \\ \mathbf{2 0 0} & \mathbf{1 0 0 . 0} & \mathbf{0 . 4 7}(\mathbf{0 . 2 3}, \mathbf{0 . 9 5})\end{array}$

$0.71(0.27,1.84)$

$1.25(0.29,5.33)$

$24.5 \quad 0.62(0.24,1.61)$

$42.90 .74(0.37,1.51)$

$100.0 \quad 0.75(0.47,1.19)$

75
197
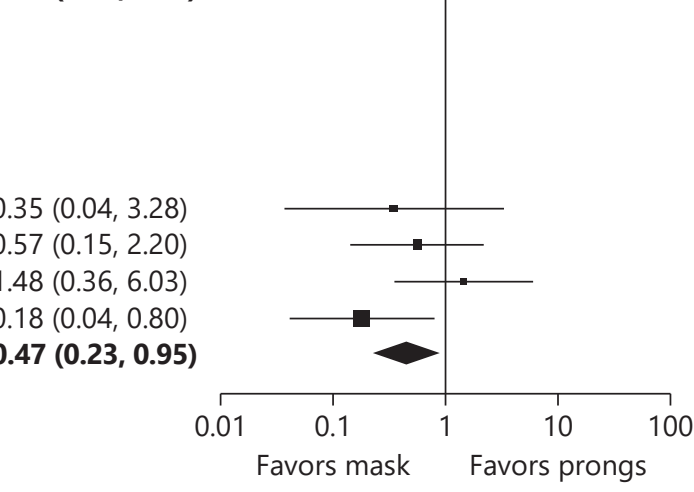

b Surfactant administration (after randomization)

Study or subgroup

Nasal mask events total

$\frac{\text { Binasal prongs }}{\text { events total }}$

$31-57$

Bashir [21], 2019

Chandrasekaran [22], 2017

Goel [1], 2015

Kieran [5], 2012

Yong [24], 2005

Total $(95 \% \mathrm{Cl})$

Total events

Heterogeneity: $\chi^{2}=11.54, \mathrm{df}=4(p=0.02) ; R^{2}=65 \%$

Test for overall effect: $Z=2.99(p=0.003)$
Weight, Risk ratio Risk ratio

$\% \quad \mathrm{M}-\mathrm{H}$, fixed, $95 \% \mathrm{Cl} \quad \mathrm{M}-\mathrm{H}$, fixed, $95 \% \mathrm{Cl}$

$\begin{array}{ll}27.7 & 0.76(0.57,1.01) \\ 11.6 & 0.28(0.11,0.67) \\ 19.8 & 1.16(0.83,1.61) \\ 11.3 & 0.62(0.35,1.09) \\ 29.6 & 0.79(0.58,1.07)\end{array}$

100.0

$0.78(0.66,0.92)$

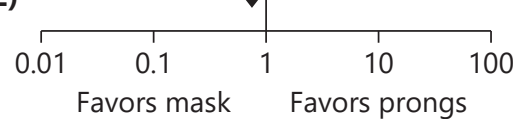

Fig. 5. Incidence of BPD and surfactant administration after randomization among patients receiving NCPAP as "step-up" therapy only. BPD, bronchopulmonary dysplasia; NCPAP, nasal continuous positive airway pressure.

ic, including 7 randomized controlled trials having enrolled a total of 722 infants. Among all infants randomized, the use of a nasal mask decreased the rate of NCPAP failure within $72 \mathrm{~h}$ of treatment initiation and the incidence of nasal injury (all grades, as well as moderate to severe only). Among a subgroup of infants requiring NCPAP for "step-up" therapy only, the use of a nasal

Nasal Mask versus Binasal Prongs in Preterm Infants mask decreased the incidence of moderate-to-severe BPD and the need for surfactant therapy.

A recent systematic review published in 2018 compared NCPAP failure and skin injury in preterm infants who received NCPAP via nasal mask versus binasal prongs [10] and noted that the use of a nasal mask reduces the risk of NCPAP and moderate-to-severe nasal 
injury. Our review adds an additional 117 patients from 1 trial published after that review [21]. Their analysis differs from ours in that they only analyzed the risk of NCPAP failure in all preterm infants and did not perform a subgroup analysis based upon the indication for CPAP. We believe patients receiving CPAP for "stepup" and "step-down" therapy can differ in several significant ways including age, severity of lung disease, type of lung disease, and comorbidities. To avoid potential confounding effects from those differences, subgroup analyses for those indications are important. As a result of the additional trial published after that review, we also found that the use of a nasal mask was associated with a significant reduction in the incidence of moderate-tosevere $\mathrm{BPD}$, an important morbidity within this population, as well as in the need for surfactant therapy. The combination of a reduction in CPAP failure, need for surfactant administration, and moderate-to-severe BPD is consistent with the picture of an overall improved respiratory status, which we believe also strengthens our conclusions as a whole.

The strengths of our review include following a rigorous review process and a systematic assessment of the included trials for risk of bias using the Cochrane riskof-bias tool [11]. We also used the GRADE criteria to assess the certainty of evidence for each outcome. We were mindful of bias during the literature review process and took steps to minimize it. To minimize the effect of missing data, we contacted each primary author of the 6 included trials and requested additional information. We also believe that our subgroup analyses, determined by the indication for CPAP, are a strength of our study (as described above) and should be included in future work.

The implications of our meta-analysis are somewhat limited due to the low number of trials identified, and the low quality of the evidence. All trials identified were at risk of performance bias due to the inability to blind the intervention, and at least at an unclear risk of detection bias. The certainty of evidence for individual outcomes was, at best, low based on the GRADE criteria. This was primarily due to the aforementioned risks of bias (primarily lack of blinding), as well as due to inconsistency of the evidence and imprecision in the estimate. Further trials are unlikely to be blinded, but they may improve the precision of the estimate and consistency of the evidence.

The generalizability of our findings has significant caveats. The trials included in this analysis mostly enrolled infants between 26 and 34 weeks of gestational age, and we cannot assume that the beneficial findings for the nasal mask extend to infants of lower gestational age or to term infants with respiratory distress. In particular, it can often be difficult to apply binasal prongs to the smallest infants $<26$ weeks. They may benefit significantly from the use of nasal masks if an adequate seal can be obtained to deliver effective CPAP and avoid mechanical ventilation. There is also variability in the type and brand of interfaces used to deliver NCPAP among the trials. The majority of the trials were conducted outside of the USA in diverse clinical settings, and it is possible that regional variations in practices and medical management could have impacted the results. Two trials included a group which rotated between patient interfaces, but 1 trial did not report respiratory outcomes, and we were unable to combine the data into a meta-analysis. Our results cannot inform clinicians about the benefit or harm from alternating NCPAP interfaces.

Despite the limitations of the currently available evidence, we believe it is important to put this evidence into the context of the previously established standard of care, which itself was based on very-low-certainty evidence. Binasal prongs were established as the standard of care based on traditional clinical practice, as well as on available evidence published by the Cochrane Collaboration in 2008 [7]. That review had identified 4 randomized trials comparing binasal prongs to other delivery interfaces among a total of 256 patients. Three of those trials addressed the efficacy of NCPAP (216 patients), but only 1 trial randomized infants immediately after delivery (36 patients).

What are the implications for practice from this systematic review? We believe that - although the evidence is of low certainty - the ease of use of nasal masks, the low technical and resource burden of transitioning from routine use of nasal prongs to nasal masks, the limited evidence supporting nasal prongs as the standard of care, and the likely minimal risk to the patient all support the use of nasal masks as the preferred interface for delivery of NCPAP. Further, well-designed clinical trials that include a more expanded population of preterm infants, to include the youngest infants who are at the highest risk of BPD and to include those infants receiving "step-down" therapy (post-extubation support), would help further strengthen the evidence and expand the population that the evidence could apply to. Lastly, a standardized assessment of nasal injury in preterm infants would enable us to more effectively compare the safety profile of the different interfaces. 


\section{Conclusions}

We found low- to very-low-certainty evidence to suggest that delivering NCPAP through the use of a nasal mask decreases the rate of NCPAP failure and the incidence of skin injury among all infants requiring NCPAP. Among preterm infants requiring NCPAP for "step-up" therapy, the use of nasal masks decreased the incidence of moderate-to-severe BPD and the need for surfactants when compared to the use of binasal prongs. There were no differences between the two interfaces with respect to mortality and other morbidities. The current evidence supports the use of nasal masks for preterm infants; however, further evidence is needed to improve the certainty of the evidence, in particular with regard to the clinically significant long-term outcome of moderate-to-severe BPD.

\section{Acknowledgements}

We would like to acknowledge and thank Amy Sisson, MS MLS, a liaison librarian at the Texas Medical Center Library for creating and conducting our search strategies within Ovid MEDLINE, Embase, Scopus, the Cochrane database, and PubMed.

\section{Statement of Ethics}

The authors have no ethical conflicts to disclose.

\section{Disclosure Statement}

The authors have no conflicts of interest to declare.

\section{Author Contributions}

B.C.K. and B.B.G. conceptualized and designed the study. They acted as independent reviewers of the titles and abstracts of the identified trials and participated in consensus meetings to determine trials' inclusion in the systematic review. They independently conducted a search for ongoing trials which met the eligibility criteria. They also independently assessed the risk of bias and GRADE of evidence and extracted demographic and outcome data from the included trials. They drafted the initial manuscript and were responsible for revisions to the manuscript.

A.J. independently reviewed the titles and abstracts of the identified trials and met for consensus meetings to determine trials' inclusion in the systematic review.

L.K., M.P., and G.S. provided methodological insight and mentoring. They reviewed drafts of the manuscript and provided comments and suggestions.

All authors approved the final manuscript as submitted and agree to be accountable for all aspects of the work.

\section{References}

1 Goel S, Mondkar J, Panchal H, Hegde D, Utture A, Manerkar S. Nasal Mask Versus Nasal Prongs for Delivering Nasal Continuous Positive Airway Pressure in Preterm Infants with Respiratory Distress: A Randomized Controlled Trial. Indian Pediatr. 2015 Dec;52(12): 1035-40.

2 Morley CJ, Davis PG, Doyle LW, Brion LP, Hascoet JM, Carlin JB; COIN Trial Investigators. Nasal CPAP or intubation at birth for very preterm infants. N Engl J Med. 2008 Feb; 358(7):700-8.

3 Davis PG, Henderson-Smart DJ. Nasal continuous positive airways pressure immediately after extubation for preventing morbidity in preterm infants. Cochrane Database Syst Rev. 2003;(2):CD000143.

4 Finer NN, Carlo WA, Walsh MC, Rich W, Gantz MG, Laptook AR, et al.; SUPPORT Study Group of the Eunice Kennedy Shriver NICHD Neonatal Research Network. Early CPAP versus surfactant in extremely preterm infants. N Engl J Med. 2010 May;362(21): 1970-9.

5 Kieran EA, Twomey AR, Molloy EJ, Murphy JF, O'Donnell CP. Randomized trial of prongs or mask for nasal continuous positive airway pressure in preterm infants. Pediatrics. 2012 Nov;130(5):e1170-6.

Nasal Mask versus Binasal Prongs in Preterm Infants
6 Davis P, Davies M, Faber B. A randomised controlled trial of two methods of delivering nasal continuous positive airway pressure after extubation to infants weighing less than $1,000 \mathrm{~g}$ : binasal (Hudson) versus single nasal prongs. Arch Dis Child Fetal Neonatal Ed. 2001 Sep;85(2):F82-5.

7 De Paoli AG, Davis PG, Faber B, Morley CJ. Devices and pressure sources for administration of nasal continuous positive airway pressure (NCPAP) in preterm neonates. Cochrane Database Syst Rev. 2008 Jan;(1): CD002977.

8 Say B, Kanmaz Kutman HG, Oguz SS, Oncel MY, Arayici S, Canpolat FE, et al. Binasal Prong versus Nasal Mask for Applying CPAP to Preterm Infants: A Randomized Controlled Trial. Neonatology. 2016;109(4):258-64.

9 Imbulana DI, Manley BJ, Dawson JA, Davis PG, Owen LS. Nasal injury in preterm infants receiving non-invasive respiratory support: a systematic review. Arch Dis Child Fetal Neonatal Ed. 2018 Jan;103(1):F29-35.

10 Jasani B, Ismail A, Rao S, Patole S. Effectiveness and safety of nasal mask versus binasal prongs for providing continuous positive airway pressure in preterm infants - a systematic review and meta-analysis. Pediatr Pulmonol. 2018 Jul;53(7):987-92.
11 Higgins J, Green S. Cochrane Handbook for Systematic Reviews of Interventions Version 5.1.0 [Internet] [updated March 2011]. The Cochrane Collaboration; 2011. Available from: www.cochrane-handbook.org.

12 Hultcrantz M, Rind D, Akl EA, Treweek S, Mustafa RA, Iorio A, et al. The GRADE Working Group clarifies the construct of certainty of evidence. J Clin Epidemiol. $2017 \mathrm{Jul}$; 87:4-13.

13 Schunemann H, Brozek J, Guyatt G, Oxman AE; GRADE Working Group. GRADE Handbook for Grading Quality of Evidence and Strength of Recommendations [Internet] [updated October 2013]. Available from: https://gdt.gradepro.org/app/handbook/ handbook.html.

14 Aydon L, Forman T, Kok C. Clustering care: using a care bundle approach to reduce adverse effects of treatment with NCPAP in the neonatal population less than 32 weeks gestation [poster presentation]. Perinatal Society of Australia and New Zealand; 2014.

15 Chawla D. Optimizing Nasal Interface for Continuous Positive Airway Pressure in Neonates. Indian Pediatr. 2015 Dec;52(12): $1027-8$.

Preterm Infants 
16 Jackson HD, Lim K, Gale TJ, Wheeler KI, Dargaville PA. Mask versus prongs for CPAP delivery: incidence of bradycardia, apnoea and desaturation [poster presentation]. Perinatal Society of Australia and New Zealand; 2013.

17 Donaldsson S, Drevhammar T, Taittonen L, Klemming S, Jonsson B. Initial stabilisation of preterm infants: a new resuscitation system with low imposed work of breathing for use with face mask or nasal prongs. Arch Dis Child Fetal Neonatal Ed. 2017 May;102(3): F203-7.

18 Szymczak A, Bokiniec R, Kornacka MK. [Assessment of the appearance of local complications associated with the use of different noninvasive ventilation techniques in neonates]. Pediatr Pol. 2013;88:405-12.
19 Arsan S. Efficacy and Safety of Nasal Mask and Prong Use in Non-invasive Ventilation for Newborns [Internet]. US National Library of Medicine, Clinical Trials Registry Trial registered 2013. ID: NCT01989442.

20 Mitchell S. Randomised trial of nasal prongs versus nasal mask for the avoidance of nasal trauma with prolonged nasal continuous positive airway pressure (NCPAP) using the infant flow driver in infants 27 weeks gestation. ISRCTN Registry Trial registered 2007. ID: 62827057.

21 Bashir T, Murki S, Kiran S, Reddy VK, Oleti TP. 'Nasal mask' in comparison with 'nasal prongs' or 'rotation of nasal mask with nasal prongs' reduce the incidence of nasal injury in preterm neonates supported on nasal continuous positive airway pressure (nCPAP): a randomized controlled trial. PLoS One. 2019 Jan;14(1):e0211476.
22 Chandrasekaran A, Thukral A, Jeeva Sankar M, Agarwal R, Paul VK, Deorari AK. Nasal masks or binasal prongs for delivering continuous positive airway pressure in preterm neonates - a randomised trial. Eur J Pediatr. 2017 Mar;176(3):379-86.

23 Newnam KM, McGrath JM, Salyer J, Estes T, Jallo N, Bass WT. A comparative effectiveness study of continuous positive airway pressurerelated skin breakdown when using different nasal interfaces in the extremely low birth weight neonate. Appl Nurs Res. 2015 Feb; 28(1):36-41.

24 Yong SC, Chen SJ, Boo NY. Incidence of nasal trauma associated with nasal prong versus nasal mask during continuous positive airway pressure treatment in very low birthweight infants: a randomised control study. Arch Dis Child Fetal Neonatal Ed. 2005 Nov;90(6): F480-3. 